\section{Migração, trabalho na indústria petroquímica e consumo de bebidas alcoólicas}

\section{Recent migration, petrochemical industry jobs and alcohol consumption}

\author{
Vilma S. Santana \\ Instituto de Saúde Coletiva \\ Universidade Federal da Bahia (UFBA) \\ Rua Padre Feijó, n. ${ }^{\circ} 29,4{ }^{\circ}$ andar - Canela \\ 40210-170 Salvador, BA - Brasil \\ e-mail:vilma@ufba.br

\section{Mauro Khoury} \\ Bolsista de Aperfeiçoamento CNPq \\ Instituto de Saúde Coletiva/UFBA

\section{Charles de Andrade} \\ Bolsistas de Iniciação Científica CNPq \\ Instituto de Saúde Coletiva/UFBA.
}

\section{Agnese Novato}

Bolsistas de Iniciação Científica CNPq Instituto de Saúde Coletiva/UFBA.

\section{Naomar de Almeida Filho}

Instituto de Saúde Coletiva / UFBA

\section{Resumo}

Objetivou-se testar testar a hipótese de uma associação entre migração recente, trabalho na indústria petroquímica e o consumo excessivo de bebidas alcoólicas, avaliando o papel de variáveis socioeconômicas e demográficas como confundidores ou modificadores de efeito. Foi empregada uma amostra aleatória de 452 chefes de família entre 18 e 60 anos de idade, proveniente de um inquérito de saúde conduzido com famílias que residiam em uma área industrial da Região Metropolitana de Salvador, Bahia, Brasil. Apenas os trabalhadores do setor industrial foram incluídos na análise $(n=299)$. Consumidores de bebidas alcoólicas foram identificados com o Questionário de Morbidade Psiquiátrica de Adultos, QMPA, aplicado por entrevistadores treinados a informantes chave de cada família selecionada. Migrantes recentes foram definidos como aqueles que tinham até dois anos de residência no local. A prevalência de consumo exagerado de álcool foi maior entre trabalhadores da indústria petroquímica e entre migrantes recentes, em comparação aos trabalhadores de outras indústrias e não migrantes recentes, respectivamente. Petroquímicos migrantes recentes apresentaram uma prevalência de consumo de bebidas alcoólicas 3,4 vezes superior ao grupo de referência. Migração recente e trabalho na indústria petroquímica são fatores de risco potenciais para o consumo de bebidas alcoólicas, por um efeito sinérgico entre essas duas variáveis. Os resultados sugerem a escolaridade como fator de proteção para o consumo de álcool, apenas entre não migrantes recentes nessa população de estudo.

Palavras chave: Migração. Trabalhadores. Indústria petroquímica. Alcoolismo. Fatores socioeconômicos. 
Abstract

This study is aimed at to evaluate the hypothesis that recent migration and work in the petrochemical industry are associated with heavy alcohol consumption, and that education and family income are relevant effect modifiers of this association. The study was based on a random sample of 452 head of families, from 18-60 years of age, drawn from a household health survey conducted in an industrial area of Bahia, Brazil. Only those who reported to have a job in the industry were eligible for this study ( $n=299$ ). Individuals with heavy alcohol consumption were identified based on the Questionário de Morbidade Psiquiátrica de Adultos (Questionnaire of Adult Psychiatric Morbidity), QMPA, completed by trained interviewers with key-informants in each family. Recent migrants were defined as those with less than two years of residency at the research site. The prevalence of alcohol abuse was significantly higher among petrochemical workers and recent migrants. The subgroup of recent migrant petrochemical workers had a prevalence 3.4 times higher than the comparison group of workers. Both recent migration and petrochemical jobs are potential risk factors for alcohol abuse. Apparently, there is a synergistic effect between these two variables for alcohol abuse. Results suggested that education may be protective only among non-recent migrants.

Keywords: Migration. Workers. Petrochemical industry. Alcoholism. Socioeconomic factors.

\section{Introdução}

Empreendimentos estratégicos, como por exemplo a construção de usinas hidrelétricas ou a instalação de complexos industriais de grande porte, ao produzirem uma demanda por força de trabalho temporária, constituem-se em importantes fatores de indução de fluxos migratórios no mundo contemporâneo ${ }^{1,2}$. O fenômeno da migração corresponde a dois importantes aspectos: por um lado, trata-se de um processo de desenraizamento social e psicológico de maior ou menor intensidade, a depender do grau de distanciamento cultural do migrante em relação ao contexto de recepção ${ }^{3-5}$. Por outro lado, pode implicar em um processo particular de seleção-exclusão do mercado de trabalho, no qual trabalhadores migrantes são destinados a ocupações de baixa qualificação e salários reduzidos. Em ambos os casos, mudanças mais ou menos forçadas de local de residência envolvem a necessidade de mecanismos psicológicos e comportamentais adaptativos potencialmente causadores ou mediadores de estresse ou outros transtornos psicopatológicos, mais comuns especialmente nos primeiros anos após a migração ${ }^{6-9}$.

Vários estudos têm revelado uma associação entre ocorrência de transtornos ou sintomas psicológicos e exposição a certos ambientes de trabalho ${ }^{10}$. Um caso especial é a indústria química, onde a ameaça de explosões, vazamento de gases e incêndios determinou a sua classificação pelo Ministério do Trabalho como Atividade Perigosa, e que exige medidas especiais de proteção ao trabalhador ${ }^{11}$. É previsível que o "estar" em ambientes de indústrias deste tipo, além das exposições químicas, possa também determinar estresse psíquico e distúrbios da conduta, como o abuso de bebidas alcoólicas. Isto poderia significar uma defesa comportamental perante o perigo latente, produzindo-se uma intoxicação alcoólica que amorteceria o estresse ${ }^{12}$, hipótese não confirmada em estudos empíricos ${ }^{13}$

Existem controvérsias sobre a relação entre exposição a atividades perigosas e abuso de álcool. Resultados de pesquisas 
mostram que bombeiros ${ }^{14}$ e policiais $^{15}$ apresentam maiores prevalências de consumo pesado de bebidas alcoólicas do que a população geral. Todavia, Leigh ${ }^{16}$, estudando empregados em ocupações perigosas, definidas como as que apresentam alta mortalidade e incidência de acidentes, encontrou apenas uma fraca associação positiva com o consumo de bebidas alcoólicas. Não foram encontrados estudos específicos sobre esta temática no setor produtivo da indústria petroquímica que focalizassem as interfaces entre as condições de vida e condições de trabalho.

No presente estudo, utiliza-se a base de dados de um estudo de morbidade geral conduzido no município de Camaçari, Bahia, durante a implantação de um pólo petroquímico regional, para testar a hipótese de que a migração recente e o trabalho na indústria química constituem fatores de risco para o consumo de bebidas alcoólicas. Avaliam-se, também, as interações das associações em pauta com covariáveis socioeconômicas, como a idade, nível de educação formal e de renda familiar.

\section{Material e Métodos}

\section{Contexto da pesquisa}

No final da década de 70, foi iniciada a implantação de um Pólo Petroquímico em Camaçari, um dos 13 municípios que integram a Região Metropolitana de Salvador. Trabalhadores de outras regiões do país foram atraídos em massa pela possibilidade de emprego, tanto nos canteiros de obras das plantas industriais quanto nas empresas de prestação de serviços às indústrias recém-instaladas. Como resultado dessa migração, a população do município triplicou em apenas dez anos, obrigando a prefeitura local a montar um programa de acolhimento, baseado na oferta de lotes urbanos conectados à rede de abastecimento de água e energia elétrica, provendo, ainda, acesso aos serviços municipais de educação e saúde ${ }^{17}$. Após as etapas iniciais de implantação, muitos dos migrantes foram absorvidos pelas próprias indús- trias do Pólo, empresas caracterizadas pelo grande porte, alta complexidade tecnológica e riscos ocupacionais e ambientais diferenciados.

\section{Amostra e coleta de dados}

Os dados analisados foram obtidos em um inquérito domiciliar realizado em 1982, com uma amostra aleatória de 510 residências da área urbana da sede de Camaçari, cujo propósito era o diagnóstico das condições de vida e saúde da população para os setores de planejamento local ${ }^{17}$. A população desse estudo é composta por todos os indivíduos do sexo masculino, de idade entre 18 e 60 anos, referidos pelos seus familiares como chefes de família. Com mapas baseados em fotografias aéreas delinearam-se sub-áreas, que foram sorteadas para inclusão no estudo. Todos os domicílios selecionados foram visitados por entrevistadores treinados, em sua maioria estudantes de medicina. Após a apresentação e consentimento em participar da pesquisa, identificava-se um membro da família, geralmente a dona de casa, como informante qualificado.

\section{Instrumentos}

Dados sociodemográficos e familiares para cada indivíduo eram registrados em uma Ficha Familiar. Todos os chefes das famílias foram entrevistados individualmente para preenchimento da História Migratória e Ocupacional-HMO, que incluía perguntas sobre o tipo e época de cada uma das ocupações e empregos anteriores. Cada informante da família também fornecia dados sobre a ocorrência de sintomas psicológicos nos membros da família, respondendo ao Questionário de Morbidade Psiquiátrica de Adultos - QMPA, instrumento originalmente desenvolvido por Santana ${ }^{18}$, e que tem sido amplamente utilizado em pesquisas populacionais sobre distúrbios mentais. Suas principais vantagens são simplicidade e rapidez na aplicação, possibilitada pelo uso de um informante secundário e não necessariamente a pessoa índex, nem sempre encontrada durante as visitas domiciliares. Acrescente-se a isso, sua adequação à lin- 
guagem coloquial empregada pela população. Estudos de validade e confiabilidade do QMPA têm sido realizados em diferentes contextos de investigação $0^{6,18,19}$. Embora o seu uso seja recomendado como etapa preliminar de desenhos de dupla fase para a detecção de casos, dados sobre as respostas a cada uma de suas questões ou escalas específicas podem se constituir em variáveis reveladoras do perfil populacional da sintomatologia psíquica, fornecendo elementos para a compreensão das demandas por ações de saúde mental.

\section{Variáveis do estudo}

A variável dependente do estudo foi "referência ao consumo excessivo de bebidas alcoólicas" (RBEX), tomada como as respostas dadas a uma das questões do QMPA diretamente relacionada à percepção de uso "excessivo" de bebidas alcoólicas - “... alguém da sua família bebe exageradamente?". Como as demais perguntas do QMPA, as respostas foram classificadas e codificadas como sim (1) e não (0). Com base na informação sobre o tempo de residência no local em anos, definiu-se a variável migração recente, que inclui pessoas nascidas em outra localidade e que ali residiam há menos de dois anos. "Não migrantes recentes” representam pessoas nascidas no local ou migrantes com mais de dois anos de residência. Uma das questões da HMO referia-se especificamente ao trabalho no Pólo Petroquímico no momento da pesquisa. Para este estudo, os dados sobre o tipo de indústria foram revisados, corrigindose inconsistências com base nas respostas sobre a descrição da ocupação e o nome da empresa que constavam da HMO. Os revisores não tinham conhecimento de outros dados do indivíduo. Assim, migração recente e trabalho em indústria do Pólo foram as variáveis independentes principais. As variáveis idade (analisada como uma variável escalar de três níveis), nível de escolaridade (alto $=0$ corresponde às pessoas com pelo menos o $1^{\circ}$ grau completo e baixo=1 abaixo desse nível) e renda familiar (dicotomizada em US\$275/mês, codificada como nível alto=0 e baixo=1) fo- ram analisadas como potenciais modificadores de efeito ou confundidores, de acordo com o modelo teórico definido e os resultados preliminares da análise tabular.

\section{Análise de dados}

Razões de prevalências (RP) foram utilizadas para avaliar associações, empregando-se, para a estimação estatística, intervalos de confiança a $95 \%$ calculados com o método Base-teste ${ }^{20}$. Na análise tabular, covariáveis em cujos estratos as estimativas pontuais de associação (RP) mostravam-se com diferenças estatisticamente significantes ao nível de $\mathrm{p}=5,0 \%$ foram selecionadas como candidatas à análise de interação para a modelagem. Para a seleção das variáveis de confundimento (confounding) potenciais, verificou-se a existência de associação entre as covariáveis candidatas e as exposições entre os não casos, e simultaneamente, entre essas mesmas covariáveis e o consumo de bebidas alcoólicas entre os não expostos a cada uma das duas variáveis independentes principais. Na análise de regressão logística, investigou-se a ocorrência de interações (modelo multiplicativo) e efeitos de confundimento (confunding), simultaneamente para as variáveis do estudo, empregando-se procedimentos "backward". Foram consideradas variáveis de interação aquelas para as quais o $\chi^{2}$ correspondente às razões de verossimilhança, relativas à retirada de termos-produtos do modelo completo (que incluía educação, idade, migração recente e renda familiar per capita), alcançavam níveis de significância estatística para um $\alpha=0,20$, de acordo com Hosmer e Lemeshow $^{21}$ (1989). Variáveis de confundimento (confunding) foram aquelas cuja retirada do modelo determinava alterações significativas na magnitude das estimativas pontuais e de precisão do odds ratio correspondentes às associações principais. O Método Delta foi empregado para estimativas de intervalos de confiança para as razões de prevalência (RP), a partir de parâmetros da regressão logística ${ }^{22}$. As combinações possíveis das duas variáveis independentes principais - migração recente e trabalho no Pólo Petroquímico - foram analisadas si- 
multaneamente através da criação de variáveis de desenho para a combinação de suas categorias, tomando-se como grupo de referência não migrantes recentes que não trabalhavam no Pólo. Para o processamento e análise dos dados, utilizou-se o software SAS, versão $6.11^{23}$.

\section{Resultados}

Dos 709 indivíduos adultos do sexo masculino da amostra, foram identificados 587 chefes de família com idade entre 18 e 60 anos. Não havia registros referentes ao consumo de bebidas alcoólicas para 135 indivíduos (27\%) que foram excluídos da análise. Entre os 452 indivíduos da população de estudo, 131 (29\%) eram migrantes recentes. Apenas os empregados no setor industrial $(\mathrm{n}=299)$ foram considerados para a análise. Cerca de metade desses trabalhadores (153/ 299, 51,2\%) referiram vinculação com o Pólo Petroquímico de Camaçari.

Na Tabela 1 são mostradas as características da população de estudo de acordo com a migração. Evidencia-se que os migrantes recentes eram relativamente mais jovens, possuíam melhor nível de escolaridade e compunham-se de maior proporção de trabalhadores do Pólo do que os "não migran- tes recentes". Estas diferenças foram estatisticamente significantes a um nível de 5\%. Não houve diferença relevante de renda familiar entre migrantes recentes e não migrantes recentes. Na Tabela 2, estão mostradas as características da população de acordo com o trabalho no Pólo Petroquímico. Verificou-se que os trabalhadores desse complexo industrial eram mais jovens, tinham melhor nível de escolaridade, maior proporção de contratados e de migrantes recentes do que os trabalhadores de outras indústrias, diferenças estatisticamente significantes a um nível de 5,0\%. Não foi observada diferença entre o nível de renda familiar para os grupos em consideração. Observou-se que migração recente estava positivamente associada com a referência a consumo excessivo de bebidas alcoólicas (Razão de Prevalência RP = 2,00; Intervalo de Confiança de 95\% IC : 1,21-3,34) e que embora os trabalhadores das indústrias do Pólo Petroquímico apresentassem prevalência referida de consumo excessivo de bebidas alcoólicas maior do que os demais trabalhadores da indústria, essa diferença não alcançou níveis de significância estatística (RP=1,25; 95\%IC: 0,68-2,30).

Os resultados da análise de regressão logística para o modelo final são mostra-

Tabela 1 - Características sócio-demográficas de acordo com migração recente (< de 2 anos) na população do estudo $(n=452)$.

Table 1 - Socio-demographic characteristics according to recent migration ( $<2$ years) in the population studied $(n=452)$.

\begin{tabular}{|c|c|c|c|c|}
\hline \multirow{2}{*}{ Variáveis sócio-demográficas } & \multicolumn{2}{|c|}{ Migração recente } & \multicolumn{2}{|c|}{ Não migrante } \\
\hline & $\mathrm{n}=131$ & $\%$ & $\mathrm{n}=321$ & $\%$ \\
\hline \multicolumn{5}{|l|}{ Idade em anos } \\
\hline $18-25$ & 34 & 26,0 & 94 & $29,3^{* *}$ \\
\hline $26-35$ & 67 & 51,2 & 117 & 36,5 \\
\hline $36-60$ & 30 & 22,9 & 110 & 34,3 \\
\hline \multicolumn{5}{|l|}{ Escolaridade } \\
\hline analfabeto / elementar & 70 & 53,4 & 209 & $65,1^{*}$ \\
\hline > elementar & 61 & 46,6 & 112 & 34,9 \\
\hline \multicolumn{5}{|l|}{ Renda familiar } \\
\hline$<$ US\$ 266,00 & 89 & 67,9 & 190 & 59,2 \\
\hline$>$ US\$265,00 & 42 & 32,1 & 131 & 40,8 \\
\hline \multicolumn{5}{|l|}{ Trabalho na Indústria ${ }^{1}$} \\
\hline Pólo & 65 & 42,5 & 88 & $19,7^{* * *}$ \\
\hline Não Pólo & 29 & 57,5 & 118 & 80,3 \\
\hline \multicolumn{5}{|c|}{$\begin{array}{l}=\text { Número de indivíduos, }{ }^{*} \mathrm{p}<0,05 ;{ }^{* *} \mathrm{p}<0,01 ; * * * \mathrm{p}<0,001 \text {. } \\
\text { - Totais diferem devido a dados não disponíveis. }\end{array}$} \\
\hline $\begin{array}{l}=\text { Number of subjects, }{ }^{*} \mathrm{p}<0.05 ; * * \mathrm{p}<0 \\
\text { Different totals because of non availabl }\end{array}$ & 0.001 & & & \\
\hline
\end{tabular}


Tabela 2 - Características sócio-demográficas de acordo com o tipo de indústria na população do estudo $(n=299)$.

Table 2 - Socio-demographic characteristics according to industry in the population studied $(n=299)$

\begin{tabular}{|c|c|c|c|c|}
\hline \multirow{2}{*}{ Variáveis sócio-demográficas } & \multicolumn{2}{|c|}{ Trabalho no Pólo Petroquímico } & \multicolumn{2}{|c|}{ Trabalho em outras Indústrias } \\
\hline & $n=153$ & $\%$ & $n=146$ & $\%$ \\
\hline \multicolumn{5}{|l|}{ Idade em anos } \\
\hline $18-25$ & 26 & 17,0 & 14 & $9,6 * *$ \\
\hline $26-35$ & 82 & 53,6 & 64 & 43,8 \\
\hline $36-60$ & 45 & 29,4 & 68 & 46,6 \\
\hline \multicolumn{5}{|l|}{ Escolaridade } \\
\hline analfabeto / elementar & 85 & 55,6 & 104 & $71,4 * *$ \\
\hline > elementar & 68 & 44,4 & 42 & 28,6 \\
\hline \multicolumn{5}{|l|}{ Renda familiar } \\
\hline$<$ US\$266,00 & 86 & 56,2 & 94 & 35,6 \\
\hline$>$ US\$265,00 & 67 & 43,8 & 52 & 64,4 \\
\hline \multicolumn{5}{|l|}{ Inserção Produção1 } \\
\hline Formal & 148 & 98,0 & 105 & $71,9 *$ \\
\hline Informal & 3 & 2,0 & 41 & 28,1 \\
\hline \multicolumn{5}{|l|}{ Migração recente } \\
\hline Sim & 65 & 42,5 & 29 & $19,9 * * *$ \\
\hline Não & 88 & 57,5 & 117 & 80,1 \\
\hline
\end{tabular}

$\mathrm{n}=$ número de indivíduos; ${ }^{*} \mathrm{p}<0,05 ;{ }^{* *} \mathrm{p}<0,01 ;{ }^{* * *} \mathrm{p}<0,001$;

1 - totais diferem devido a dados não disponíveis.

$\mathrm{n}=$ Number of subjects, ${ }^{*} \mathrm{p}<0.05 ;{ }^{* *} \mathrm{p}<0.01 ;{ }^{* * *} \mathrm{p}<0.001$

1 - Different totals because of non available data.

Tabela 3 - Razões de Prevalência e respectivos Intervalos de Confiança obtidos com os parâmetros da regressão logística para a associação entre migração recente e trabalho em indústrias do Pólo Petroquímico de Camaçari e Referência de Consumo Excessivo de Bebidas Alcoólicas (RBEX), ajustadas para a Idade ( $\mathrm{n}=299$ ).

Table 3 - Reasons for prevalence and their respective reliability ranges obtained with logarithmic regression parameters for the association between recent migration and work in the Camaçari Petrochemical Complex and reference of excessive consumption of alcohol (RBEX), age-adjusted $(n=299)$.

\begin{tabular}{|c|c|c|c|}
\hline Variáveis incluídas no modelo final & $\begin{array}{l}\text { Razão de Prevalência para } \\
\text { a associação } \\
\text { com RBEX }\end{array}$ & \multicolumn{2}{|c|}{$\begin{array}{c}\text { Intervalo } \\
\text { de Confiança }{ }^{1} \text { de } 95 \%\end{array}$} \\
\hline \multicolumn{4}{|l|}{ Educação < Elementar ( n=189) } \\
\hline Não migrante/ Não Trabalhador do Pólo & 1 & - & - \\
\hline Migrante / Trabalhador do Pólo & 3,43 & 1,19 & 9,95 \\
\hline Migrante /Não Trabalhador do Pólo & 2,91 & 0,72 & 11,80 \\
\hline Não Migrante / Trabalhador do Pólo & 1,24 & 0,39 & 3,88 \\
\hline Idade $^{2}$ & 2,16 & 1,03 & 4,50 \\
\hline \multicolumn{4}{|l|}{ Educação > Elementar ( $n=110)$} \\
\hline Não migrante/ Não Trabalhador do Pólo & 1 & - & - \\
\hline Migrante / Trabalhador do Pólo & 7,07 & 0,82 & 60,88 \\
\hline Migrante / Não Trabalhador do Pólo & 5,74 & 0,59 & 55,21 \\
\hline Não Migrante / Trabalhador do Pólo & 1,51 & 0,13 & 17,18 \\
\hline Idade $^{2}$ & 2,20 & 0,73 & 6,65 \\
\hline \multicolumn{4}{|c|}{$\begin{array}{l}\text { RBEX - Referência a Consumo Excessivo de bebidas alcoólicas; } \\
\text { n = subtotal da população do estudo; } \\
1 \text { - Estimado através do Método Delta; } \\
2 \text { - Idade foi incluída no modelo como variável escalar }(1,2,3) \text {, as demais codificadas como } 1 \text { e } 0 \text {. }\end{array}$} \\
\hline \multicolumn{4}{|c|}{$\begin{array}{l}\text { RBEX - Reference of excessive consumption of alcohol. } \\
\mathrm{n} \text { - Subtotal in the population studied } \\
1 \text { - Estimated through the Delta Method. } \\
2 \text { - Age was included in the model as a scaled variable }(1,2,\end{array}$} \\
\hline
\end{tabular}


dos na Tabela 3, especificando-se as razões de prevalência para a associação de referência a consumo excessivo e cada um dos grupos de combinação das categorias das variáveis independentes principais, i.e., migração recente e trabalho no Pólo Petroquímico. Como a variável educação revelou-se como modificadora de efeito (Razão de Máxima Verossimilhança = 4,32, para 1 grau de liberdade) desenvolveramse modelos para cada um dos estratos dessa variável. Idade foi mantida em ambos os modelos por ser variável confundidora. O principal achado é que entre os indivíduos de educação menor do que elementar, migrantes recentes trabalhadores do Pólo apresentaram prevalência referida de consumo excessivo de álcool mais de três vezes maior do que o grupo de referência (RP ajustada pela idade $=3,43$; 95\% IC: 1,19-9,95). Entretanto, a migração recente ou o trabalho no Pólo, isoladamente, não se constituíram per se em fatores de risco potenciais para o consumo excessivo de bebidas alcoólicas, indicando, neste estudo, um sinergismo entre essas duas variáveis. Entre os trabalhadores de educação acima da elementar, embora as razões de prevalência apresentem tendência semelhante às do grupo descrito anteriormente, não houve diferenças estatis-

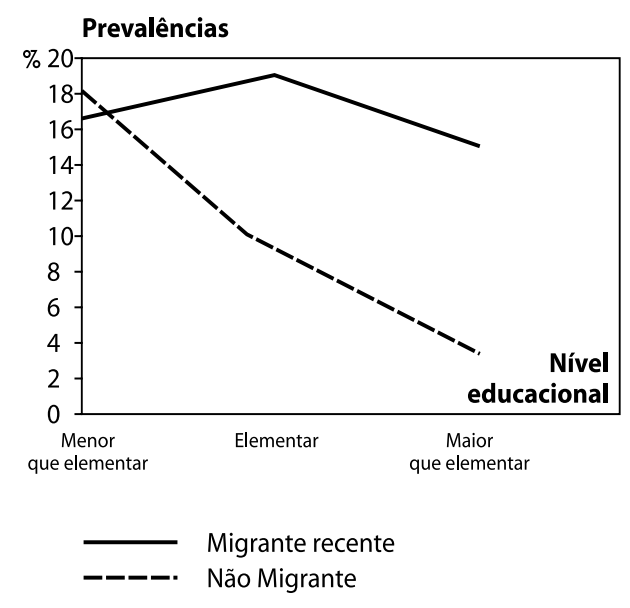

Figura 1 - Prevalência do consumo excessivo de álcool de acordo com a escolaridade e migração recente.

Figure 1 - Prevalence of excessive consumption of alcohol according to education and recent migration. ticamente significantes. Nesse último grupo, os intervalos de confiança alargados são evidência do insuficiente poder do estudo para essa análise.

Para visualizar em maior detalhe os resultados da complexa relação entre nível de educação, migração recente, trabalho no Pólo Petroquímico e consumo de bebidas alcoólicas, elaboraram-se os gráficos mostrados nas Figuras 1 e 2. Nota-se que as curvas de prevalência referida de consumo excessivo de álcool por nível de escolaridade (definida em três níveis, analfabetos, elementar e mais que elementar) são distintas para migrantes recentes e não migrantes recentes. A freqüência de consumidores de bebidas alcoólicas é maior entre migrantes recentes do que nos não migrantes recentes independente do nível de escolaridade, exceção para o grupo de analfabetos. Todavia, enquanto o consumo de bebidas alcoólicas se reduz acentuadamente com o aumento do nível de escolaridade entre os não migrantes, distintamente, entre os migrantes recentes a prevalência se mantém estável em torno de $17 \%$ (Figura 1). Em relação à outra variável independente, trabalho no Pólo Petroquímico, embora a análise tenha sido prejudicada pelo pequeno número da amostra, verifica-se que também a prevalência re-

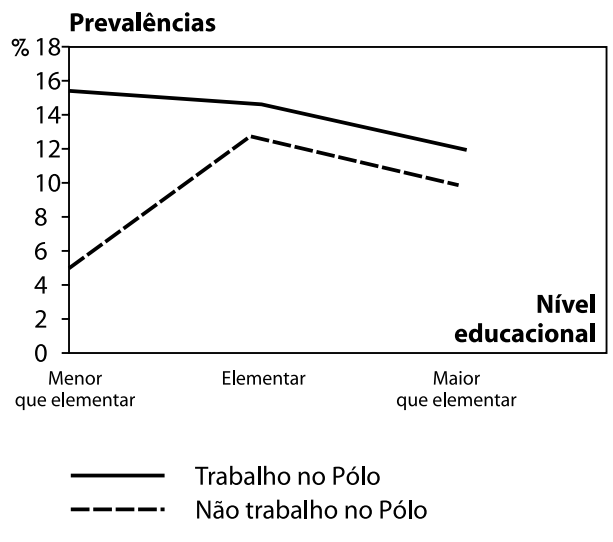

Figura 2 - Prevalência do consumo excessivo de álcool de acordo com a escolaridade e trabalho no pólo petroquímico.

Figure 2 - Prevalence of excessive consumption of alcohol according to education and work in the Petrochemical Complex. 
ferida de consumo excessivo de bebidas alcoólicas é maior entre os empregados do Pólo do que nos trabalhadores de outras indústrias, em todos os níveis de escolaridade considerados, especialmente quando o grupo é de analfabetos (Figura 2).

\section{Discussão}

Os resultados desta investigação indicam uma complexa interrelação de fatores como migração recente, trabalho no Pólo Petroquímico, escolaridade e idade com o padrão de consumo de bebidas alcoólicas. Embora a migração recente e o trabalho no Pólo não tenham sido reconhecidos como potenciais fatores de risco isolados, essas duas condições parecem atuar sinergicamente, potencializando os seus efeitos recíprocos em relação ao padrão de consumo de bebidas alcoólicas. Isto ocorre para os indivíduos de escolaridade igual ou menor que elementar, mesmo após o ajuste por idade, identificada como um fator de risco para a referência a consumo excessivo de bebidas alcoólicas, também observada em associação com o trabalho nas indústrias do Pólo. Em outras palavras, os trabalhadores que haviam migrado há menos de dois anos para a área do estudo, e simultaneamente trabalhavam em indústrias do Pólo Petroquímico, apresentavam uma prevalência de consumo de bebidas alcoólicas mais do que o dobro da estimativa entre os nascidos no local ou que migraram há mais de dois anos e trabalhavam em outro tipo de indústria. Esses resultados são estatisticamente significantes entre os indivíduos de menor nível de escolaridade, e não se modificam quando se ajusta para um possível efeito confundidor da idade. Ademais, observou-se também que a prevalência de consumo referido de bebidas alcoólicas apresentava-se em associação inversa com a escolaridade apenas entre os não migrantes recentes. Portanto, esses achados apontam para uma estreita relação entre as condições de trabalho e as condições de vida e saúde, e conseqüentemente para as limitações de abordagens simplistas das questões referentes à origem ocupacional de agravos para a saúde, ao considerar os possíveis fatores de risco isoladamente e não na complexidade de suas relações. Revela-se aqui a importância de outros aspectos, socialmente determinados, da vida dos trabalhadores como nível de escolaridade e situação de desterritorialização recente, em conjunção com um certo tipo de trabalho no setor industrial, que expressam a complexidade dessas relações não visíveis nos parâmetros da modelagem, mas requerem uma combinação de procedimentos gráficos descritivos para a sua melhor compreensão.

No mundo contemporâneo, o processo de modernização acelerada das sociedades tem se configurado basicamente através da internacionalização da produção, resultando no que se tem denominado de globalização da economia ${ }^{1,24}$. Na esfera dos processos sociais, dentre as consequências destas transformações, destacam-se o aumento do desemprego estrutural devido à automação industrial e a desterritorialização da força de trabalho, com intensificação de correntes migratórias internas e externas ${ }^{2,3}$. Os mecanismos de seleção, terceirização e, principalmente para o objeto desse estudo, o deslocamento da mão de obra, resultam em perfis epidemiológicos alterados, especialmente na esfera da saúde psíquica ${ }^{25}$. Estudos epidemiológicos sobre as relações entre migração, mudança cultural e morbidade psicossocial foram conduzidos no Peru $^{4,26}$ e na Colômbia ${ }^{27}$, revelando, consistentemente, um efeito deletério do deslocamento forçado sobre a saúde psicológica dos indivíduos. No Brasil, incidentalmente, a maioria dos estudos populacionais sobre essa temática foi realizada no Estado da Bahia. Almeida-Filho e Bastos ${ }^{6}$ (1982), em um estudo de caso-controle, encontraram um importante efeito da migração sobre estados depressivos em mulheres. Santana ${ }^{18}$ (1982), em um estudo de morbidade mental com 1549 indivíduos, encontrou prevalência maior entre migrantes do que em não-migrantes. Esta hipótese foi estudada por Almeida-Filho ${ }^{28}$ (1985) que, levando em consideração os efeitos de gênero, idade, situação conjugal, educação, renda e tama- 
nho da família, verificou uma interação entre a experiência migratória e a inserçãoexclusão do mercado de trabalho para escores elevados de sintomas mentais. Mais recentemente, com a base de dados do Estudo Nacional Multicêntrico de Morbidade Mental $^{19}$, encontraram-se também evidências de associação positiva entre morbidade psiquiátrica menor e migração, que se elevava entre mulheres migrantes desempregadas, quando comparadas àquelas que tinham uma atividade remunerada fora da residência ${ }^{8}$. Relatos de trabalhadores do Pólo Petroquímico de Camaçari apontam a ansiedade e o medo em relação aos riscos ocupacionais como problemas psicológicos comuns, que poderiam resultar em um consumo pesado de bebidas alcoólicas. Nessa direção, encontram-se os achados de Rangel ${ }^{29}$ sobre a percepção de trabalhadores do Pólo de que as suas ocupações eram de "alto risco", sendo comuns as preocupações com o estado geral de saúde.

Apesar dos avanços, as limitações dos estudos epidemiológicos impedem um maior aprofundamento das questões referentes ao significado diferencial da experiência migratória, particularmente no que diz respeito aos aspectos subjetivos do problema. Para Grimberg e Grimberg ${ }^{9}$ a migração é psicologicamente conflitiva ao implicar num processo de mudança simultânea de muitos objetos, afetando a estabilidade psíquica de modo generalizado. Este processo, manifesto na cotidianidade dos sujeitos, será influenciado pelo grau de estabilidade da estrutura psíquica e pelas situações externas a que se submete o indivíduo que, no caso do migrante, atuam especialmente nos primeiros meses de exposição ao novo contexto ${ }^{5}$. Além disso, o migrante de baixa renda geralmente sofre uma exposição mais intensa a fatores de vulnerabilidade próprios dos centros urbanos: precárias condições de moradia; barreiras de acesso a serviços de saúde, educação, lazer e serviços comunitários de modo geral; maior vulnerabilidade à violência e aos riscos de adoecer. Todos estes fatores resultariam em um aumento do estresse social e, conseqüentemente, na busca da redução do sofrimento psíquico por diversas estratégias de coping, que poderiam incluir o uso e abuso de substâncias psicotrópicas, como por exemplo o álcool. Para compreender as repercussões psicossociais da migração é necessário considerar tanto as características e experiências individuais como as conjunturas políticas, econômicas e culturais dos contextos de origem e de destino ${ }^{3}$.

Os achados do presente estudo devem ser tomados com precauções, devido às suas várias limitações. Este é um estudo transversal e a anterioridade das exposições em relação ao efeito não pode ser estabelecida. Portanto, as associações encontradas podem ser resultantes de uma dinâmica de seleção entre os grupos em análise, ou mesmo diferenciais na terminação do consumo excessivo de álcool. Embora as informações sobre o consumo de bebidas alcoólicas tenham tido a vantagem de serem obtidas de familiares e não do próprio indivíduo, que tende à negação, é clara a fragilidade da variável de efeito baseada em uma questão específica, fundada na subjetividade de uma percepção individual, demarcada na expressão- "excessivo". Todavia, estas respostas resumem um julgamento coletivo da própria família, mediada pela pessoa informante que pode ser considerada uma expressão do não-permitido ou não-aceitável.Vale ressaltar, também, que se trata de um estudo exploratório, realizado para tão-somente levantar questões de pesquisa plausíveis que, pela sua importância, estão a merecer estudos mais apropriados com medidas de efeito mais acuradas.

Uma limitação ao potencial de generalização destes achados prende-se à população do estudo. Enquanto os trabalhadores do Pólo Petroquímico residiam em várias partes da Região Metropolitana de Salvador, esta pesquisa foi conduzida apenas com aqueles que moravam no município sede de Camaçari, em uma época em que a instalação desse complexo industrial se processava. Muitas das ocupações então disponíveis representavam atividades relativas à sua construção e instalação, embora muitas de suas fábricas já se encontrassem em operação. Embora fosse notória a complexidade 
da tecnologia implantada e os altos riscos potenciais para o ambiente ou a saúde humana que elas representavam, emprego no Pólo era visto como de prestígio e fonte de valorização social e as expectativas eram de altos salários e grande impacto no desenvolvimento econômico e social da região ${ }^{17}$. O que se observa nessa pesquisa, ao contrário, é que não havia diferenças significativas nos rendimentos familiares de empregados do Pólo e os demais trabalhadores que residiam em Camaçari. As diferenças encontradas foram relativas à idade e escolaridade (trabalhadores do Pólo mais jovens e de melhor nível de educação), o que indica não um impacto social benéfico, mas sim processos de seleção efetivos para a identificação dos mais qualificados, desde que não parece plausível que anos de educação formal ou idade se modifiquem com o tipo de emprego.

Quanto à escolaridade, sabe-se que esse é um importante fator de proteção para distúrbios mentais ${ }^{30}$, ao qual se atribui uma melhor habilitação dos indivíduos nos já citados mecanismos de coping. Daí resultaria um melhor gerenciamento de conflitos interpessoais, familiares e profissionais que preservam o equilíbrio emocional e psíquico em situações de grande demanda emocional, como no período imediatamente após a migração ou nos primeiros anos de emprego em indústrias consideradas de alto risco ${ }^{31}$. A escolaridade também expressa padrões de consumo de bens materiais (nível socioeconômico), acesso a meios de qualificação pessoal e profissional, e por conseguinte, maior poder de barganha contratual no mercado de trabalho. O consumo pesado de bebidas alcoólicas é conhecido por sua associação negativa com o nível de escolaridade ${ }^{30}$, mas pouco se sabe sobre as suas interações com a situação migratória. Neste estudo, tais interações são reveladas, mostrando que mecanismos mais coletivos de coping podem estar desativados ou não são suficientes para dar conta do desconforto psíquico originado com a migração.

É possível que este cenário se aplique apenas aos trabalhadores do Pólo, moradores de Camaçari, um município com pou- cos recursos de infra-estrutura urbana e baixa atratividade para a classe média. Assim, a amostra do estudo estaria representando apenas os segmentos mais pobres e de mais baixa qualificação do quadro funcional daquelas empresas, selecionados por local de residência. Pesquisas de desenho transversal são limitadas na atribuição de relações causais por não permitirem a identificação da antecedência da exposição em relação aos efeitos. Portanto, não é possível com esses dados afirmar que o trabalho no Pólo ou a migração recente "causariam" o consumo de bebidas alcoólicas, ou se indivíduos com esse comportamento foram seletivamente atraídos para migrar e procurar trabalho nesse tipo de indústria. Contudo, desde que esses sujeitos foram submetidos a exames admissionais, é possível que ao exame clínico o consumo de bebidas alcoólicas pudesse ser detectado, especialmente pela consideração de que o trabalho no Pólo requer segurança máxima.

Com a globalização e a unificação de mercados em várias partes do mundo, e o refluxo da implantação de grandes plantas industriais nos centros urbanos em países em desenvolvimento ou em suas proximidades, verifica-se que a migração de trabalhadores se intensifica, tanto internamente no próprio país como para outros países. Esses dados alertam para a necessidade de atenção especial a esses trabalhadores, especialmente aqueles que não dispõem de nível educacional pelo menos elementar. Programas integrados que envolvam atividades de acolhimento mais abrangentes em relação às demandas psicológicas e culturais deverão fazer parte obrigatória das estratégias de recrutamento de trabalhadores migrantes.

\section{Agradecimentos}

Ao Instituto de Saúde Coletiva/UFBa que contribuiu com a infraestrutura física, cessão de equipamentos e material de escritório. Participaram também desse estudo os bolsistas de Iniciação Científica Lucas Ferreira, Adriana Matos, Adriana Andrade e a Mestranda Lúcia Carrozzo. Esta pesquisa se tornou possível com a colaboração dos moradores de Camaçari. 


\section{Summary}

This study is aimed to evaluate the hypothesis that recent migration and petrochemical industry jobs are associated with heavy alcohol consumption, and to examine whether socioeconomic and demographic variables are relevant effect modifiers or confounders for these associations. In the late 70's, a petrochemical industry complex was settled in the city of Camaçari, Metropolitan Region of Salvador, BahiaBrazil. These industries brought a flow of migrants from surrounding areas and determined deep changes in the urban structure and the local environment. To provide epidemiological data for a health monitoring system, a broad general morbidity survey was carried out with people living in the area. A random area sample of 510 households was taken from all urban domiciles. From the total of 2695 subjects, only male family heads, over 18 years of age, who reported to have a job in the industry were eligible for this study population ( $n=308)$. For those families that agreed to participate, questionnaires were completed by trained interviewers with a qualified informant, mostly housewives. Heavy alcohol drinkers were identified by family informants with a validated screening instrument, the Questionnaire of Adult Psychiatric Morbidity, QMPA. Data on individual occupational and migration background were registered. Prevalence ratios and $95 \%$ confidence intervals, as obtained by logistic regression parameters, were used to estimate the statistical associations under investigation. A total of 299 individuals comprised the study population. Petrochemical industry workers represent $51 \%$ of individuals employed in the industrial sector. Only 29\% reported to have at least two years of residency in the area. Prevalence of heavy alcohol drinking was positively associated with petrochemical industry jobs and recent migration, separately. Recent migrants who were also petrochemical workers had a prevalence estimate more than three times higher than the reference group composed by non-petrochemical non-recent migrant workers (Prevalence Rate $=3.43$; 95\% CI: 1.19, 9.95). Years of education were negatively associated with heavy alcohol use only among non-recent migrants. Petrochemical industry jobs were positively associated with heavy alcohol consumption in all ages and at any level of formal education. Although cross-sectional studies are limited for identifying risk factors, these findings are suggestive that both recent migration and petrochemical jobs are potential risk sources for heavy alcohol drinking. Apparently, there is a synergistic effect between these two variables. Education may be protective for heavy drinking, particularly among non-recent migrants. Results support recommendations for better social support for migrants who are engaged in risky jobs, such as those in the petrochemical industry.

\section{Referências}

1. El Batawi MA. Migrant workers. In: Jeyratnam J. Occupational health in developing countries. New York: Oxford University Press; 1992.

2. Recollons L. Les migraciónes actuales en el contexto de la población mundial. Rev Treball Soc 1991;124:6-45.

3. Bibeau G, Chan-Yip AM, Lock M, Rousseau C, Sterlin C, Fleury H.La santé mentale et ses visages.Québec: Gaëtan Morin; 1992.

4. Ponce OV.Migración interna a la metrópolis. Lima:Imprenta de la Universidad de San Marcos; 1970.
5. Trad LA.Procesos migratorios y salud mental: el caso de los brasileños en Barcelona. [Tese de Doutorado] Barcelona: Departamento de Sociologia y Metodologia de las Ciencias Sociales, División de Ciencias Juridicas Economicas y Sociales, Universitat de Barcelona; 1996.

6. Almeida Filho N, Bastos SB. Estudo caso-controle da associação entre migração e desordens depressivas em mulheres.J Bras Psiquiatr 1982; 31:25-30.

7. Almeida Filho N. Social epidemiology of mental disorders: a review of Latin American studies. Acta Psychiatr Scand 1987; 75: 1-10. 
8. Coutinho E, Almeida Filho N, Mari JJ, Rodrigues L. Minor psychiatric morbidity and internal migration in Brazil. Soc Psychiatr Psychiatr Epidemiol 1996; 31:173-9.

9. Grimberg M, Grimberg J.Psicopatología de la migración. Barcelona: Alianza; 1984.

10. Fletcher B.Work, stress, disease and life expectancy.New York: John Wiley \& Sons; 1991.

11. Ministério do Trabalho. Segurança e medicina do trabalho. São Paulo: Atlas; 1994. (Série Manuais de Legislação Atlas, 16).

12. Neff JA. The stress-buffering role of alcohol consumption: the importance of symptom dimension.J Hum Stress 1984; (Spring):35-42.

13. Allan CA. Alcohol problems and anxiety disorders - a critical review. Alcohol \& Alcoholism 1995;30:145-51.

14. Boxer PA, Wild D. Psychological distress and alcohol use among firefighters. Scand J Environ Health 1993; 19:121-5.

15. Violanti J, Marshall J, Howe B. Police occupational demands, psychological distress and the coping function of alcohol. J Occup Med 1983;25:455-8.

16. Leigh JP. Dangerous jobs and heavy alcohol use in two national probability samples. Alcohol \& Alcoholism 1995; 30:71-86.

17. Noronha CV, Carvalho FM. Camaçari: migração e investimentos em saúde sob a égide do capital monopolista. Rev Baiana Saúde Pública 1985; 12:29-37.

18. Santana VS. Estudo epidemiológico das doenças mentais em um bairro de Salvador. Salvador:Secretaria de Saúde do Estado da Bahia; 1982. (Série de Estudos em Saúde, 3).

19. Almeida Filho N, Mari JJ, Coutinho E, França J, Fernandes J, Andreoli S, Busnello E. Brazilian multicentric study of psychiatric morbidity - methodological features and prevalence estimates. Br J Psychiatr 1997; 171:524-9.
20. Kleinbaum D, Kupper L, Morgestern H. Epidemiologic research - principles and quantitative methods. New York: Van Morrisson \& Rostrand; 1982.

21. Hosmer DW, Lemeshow H. Applied logistic regression. New York: John Wiley \& Sons; 1989.

22. Oliveira NF, Santana VS, Lopes AA. Razões de proporções e Método Delta para intervalos de confiança. Rev Saúde Pública 1997; 31:90-9.

23. SAS Institute Inc. Statistical analysis system. Version 6 User's guide [software]. $4^{\text {th }}$ ed. Cary, NC:SAS Institute Inc.; 1989.

24. Castells M. A economia informacional: a nova divisão internacional do trabalho e o projeto socialista. Cad CRH 1992; 17:5-34.

25. Santana VS. A globalização e a saúde do trabalhador no Brasil: o que está contecendo e o que poderá acontecer. In: Anais da 4a. Reunião Especial da SBPC, 1996. Feira de Santana. Feira de Santana:UEFS/SBPC; 1996. p 41-6.

26. Caravedo B, Rotondo H. Mariátegui J.Estudios de psiquiatría social en el Peru. Lima:Ediciones del Sol; 1963.

27. Micklin M, León C. Life change and psychiatric disturbance in a South American city: effects of geographic and social mobility.J Health Soc Behav 1978; 19:92-107.

28. Almeida Filho N. Migration and mental health in Bahia. Sevilla: Caja de Ahorros de la Inmaculada; 1985.

29. Rangel ML. Saúde dos trabalhadores: um estudo das representações e da subjetividade dos trabalhadores da indústria petroquímica.Cad Saúde Pública 1993;9:333-48.

30. Dohrenwend B, Levav I, Shrout PE, Schwartz S, Naveh G, Link $B$ et al. Socioeconomic status and psychiatric disorders: the causation-selection issue. Science 1992;255:946-52.

31. Jeyratnam J.Occupational health in developing countries. London: Oxford University Press; 1992. 